\title{
Intelligent Estimator for Assessing Apple Fruit Quality
}

\author{
Ajay Pal Singh Chauhan \\ Department of ECE \\ Sant Longowal Institute of Engg.\& \\ Tech. Longowal Distt. Sangrur, Punjab(INDIA)
}

\author{
Amar Partap Singh \\ Department of ECE \\ Sant Longowal Institute of Engg.\& Tech. \\ Longowal Distt.Sangrur, Punjab(INDIA)
}

\begin{abstract}
The proposed intelligent estimator is implemented using nearest neighbor classifier for automatic grading of red delicious apple fruit from its surface color using machine vision. Though different variants of nearest neighbor classifier are reported in the literature for color classification yet no systematic study is reported till-date for its application in fruit quality assessment using surface color information. The present work reports on comparative evaluation of different variants of nearest neighbor classifier for assessing the quality of apple fruit. It has been found experimentally that amongst different variants, Euclidean Distance Metric based k-Nearest Neighbor Classifier is best suited for this particular application. The performance of this classifier is evaluated at different illuminations of the fruit surface. It is found that efficiency is the highest at a particular intensity of surface illumination. In fact, efficiency achieved using proposed estimator is nearly $95.12 \%$ if manual grading is assumed to be $100 \%$ accurate taken as reference. However, $4.88 \%$ variation is due to subjective judgment of human-beings in perceiving the apple fruit visually, which of course is obvious. Moreover, the repeatability of the proposed system is found to be $100 \%$ as observed after rigorous experimental validation.
\end{abstract}

\section{Keywords}

Intelligent, estimator, classifier, color, fruit and neighbor

\section{INTRODUCTION}

Food industry is amongst the industries that largely use machine vision for inspection of produce. Machine vision systems benefit from specially designed digital image processing software to accomplish this task. However, there is an urgent need to develop an intelligent fruit quality grader that manifests effectively human visual perception in assessing fruit quality using machine vision. In order to do so, an attempt is made in this regard for estimating fruit quality using machine learning while considering fruit surface illumination as one of the important parameters in deciding the accuracy of the proposed estimator. Fruits are graded based on their external factors like shape, size, color and external defects, etc. Color provides important information in estimating maturity and examining the freshness state of fruits. Machine vision has proved to be an excellent tool that could be very well used to replace human sorters for reliable and consistent judgment in estimating and comparing color of the fruits. Though human inspectors may make different judgments on the same product at different instances yet machine vision is free from such type of errors. Physical fatigue associated with the continuous working of human inspector is another problem that affects the performance of human inspectors adversely.
However, classification of fruits based on color is the most important tasks performed in the packaging industry. By comparing set of significant features of an unknown sample to a set of features that represent classes of known samples the classification identifies a sample. Color features are used by color classifier to identify samples based on their color. Two phases are there in classification, training phase and classifying phase. During the training phase the machine vision software is taught, the types of samples required to be classified during the classifying phase. A sample is classified by classifying phase according to how similar the sample features match features of the trained samples [1-4].

Various Color models are available for qualitative as well as quantitative evaluation of color of an object in machine vision. Color classification process involves extraction of useful information related to the spectral properties of object surfaces by discovering the best match from a set of known descriptors or class models to implement the recognition task [5][6]. Design of visual based color classification system is reported using RGB color model histograms [7] in which the color feature represents the three dimensional color information of the sample in a one dimensional format. RGB and HSL are the most commonly used color space for color grading [8]. As electronic images are often acquired in terms of RGB, so RGB is most commonly studied color space. However, due to the $3 \mathrm{D}$ representation of colors, color grading in the RGB color space is complicated making the selection and adjustment of color preferences difficult as thresholding is needed in all three color channels. Adjacent grades may correspond to regions that are not contiguous thus making it difficult for an operator to make even slight adjustments to classification threshold during production. However, most of the color grading systems use HSL rather than RGB values to specify color preferences since it is a more intuitive representation employing human distinguishable hues [9].Based on these considerations, the proposed intelligent estimator is implemented using HSL color space to calculate a color feature for every sample to be trained or classified. In fact, HSL model provides a highly effective color evaluation particularly for analyzing biological things. In biological things light varies widely as a function of wavelength.

As found experimentally HSL model is most suitable for finding out the ripeness of vegetables, fruits as intensity component is decoupled from color information. Hue value is also somewhat invariant to change in light intensity [10]. Moreover, the way in which human being perceives color, Hue and Saturation components are intimately related to that. However, disadvantage of HSL model over RGB is that set of expected color for a given application will not necessarily correspond to contiguous hue values. However, in order to circumvent this problem, the hue is normalized to lie between 0 degree and 360 degree with red represented as 0 degree, green as 120 degree and blue as 240 degree. But at the same 
time, this introduces visible discontinuities into the color space making it difficult for a user to set adjust color grade boundaries using hue values alone. Due to this reason, the present study well considers saturation component in deciding the boundaries. Moreover, by using hue for color grading alone does not contain brightness information, a dark red with low intensity can have same hue value as much as brighter red. However, this drawback can be overcome by including the intensity component from the HSL color space in the computations [9]. Due to this reason, the present study proposed to include intensity component of surface illumination as an important parameter while using HSL model for evaluation of fruit quality.

Standard database is also available for inspection of apple fruit on the basis of which the results are formulated in the present study as reference. However, different color analysis methods are reported in the literature for estimation of fruit quality on the basis of surface color including mathematical, statistical and neuro computational in particular. Study on HSL color model based fruit quality evaluation is also reported [11] in which RGB is first converted into HSL model and hue histogram was estimated using back-propagation neural network. In another work, machine vision system is designed using color space in which RGB is first converted into the YIQ [12]. Here RGB color space is separated into a luminance $\operatorname{part}(\mathrm{Y})$ and two chrominance parts $(\mathrm{Cb}$ and $\mathrm{Cr})$. Then linear transform is used to convert the RGB values into YIQ easily. Study is also available on RGB calibration for color image analysis in machine vision [13]. Here, color calibration is performed at all pixels in the image. However, the program has a windowing function to limit color calibration to a local region of interest. For finding out the ripeness of apple it has been found experimentally that HSL model is most suitable. In this work, RGB model after performing normalization, values are first converted into HSL using a set of converting equations in $\mathrm{C}++$ and then statistical analysis is applied. However, in this classification task, only one parameter (median density) of hue of the apple is used as the criterion for grading [10].In another interesting work, fusion of texture and color features for fruit recognition is also proposed in which recognition is done, derived from the wavelet transformed sub bands by the minimum distance classifier based upon statistical and co-occurrence features [14]. One more study is reported in which object classification was accomplished in the testing stages by means of discriminate analysis and k-nearest neighbor algorithms using mean standard deviation of RGB [15]. Another study is available in which using multispectral images, statistical and syntactical classifiers are trained for two and multi category grading of the fruits [16].An algorithmic model for automatic classification of different types of flowers using k-NN classifier is also proposed [17].

After having rigorous literature review, it has been observed that these methods, in fact, fail to address the effect of intensity component of fruit surface illumination. However, based on the successful results of these studies, the authors of the present paper decided to extend the concept of color classification to assess fruit quality using nearest neighbor classifier while considering intensity component as one of the important deterministic parameters. In order to do so, different variants of nearest neighbor algorithm have been evaluated under different conditions of surface illumination to find an optimal solution for the evaluation of fruit quality from its surface color.

\section{NEAREST NEIGHBOR CLASSIFICATION}

Supervised learning is the fundamental task in machine learning and nearest neighbor classification falls under this category [18].In supervised learning, training examples and test examples are there. A training example is an ordered pair $\mathrm{hn}$, pi, where $\mathrm{n}$ is an instance and $\mathrm{p}$ is a label. A test example is an instance $n$ with unknown label. The goal is to predict labels for test examples. Assume that instances $\mathrm{n}$ are members of the set $\mathrm{N}$, while labels $\mathrm{p}$ are members of the set $\mathrm{P}$. Then a classifier is any function $\mathrm{f}: \mathrm{N} \rightarrow \mathrm{P}$. Though a supervised learning algorithm is not a classifier itself, yet it is an algorithm whose output is a classifier. Mathematically, a supervised learning algorithm is a higher-order function of the type $(\mathrm{N} \times \mathrm{P}), \mathrm{r} \rightarrow(\mathrm{N} \rightarrow \mathrm{P})$, where $\mathrm{r}$ is the cardinality of the training set. The most intuitive way of determining the class of a feature vector is to find its proximity to a class or features of a class using a distance function. For calculating distance of an input vector from the unknown class, three different Distance Metrics [2] as used are given below:

Let $\mathrm{M}=\left[m_{1}, m_{2}, \ldots m_{n}\right]$ and $S=\left[\mathrm{s}_{l}, s_{2}, \ldots s_{n}\right]$ be the feature vectors, then

$$
\begin{aligned}
& \text { Euclidean Distance, } d(M, S)=\sqrt{\sum_{i=1}^{n}\left(M_{i}-S_{i}\right)^{2}} \\
& \text { Sum Distance, } d(M, S)=\sum_{i=1}^{n}|M i-S i|
\end{aligned}
$$

$$
\text { Maximum Distance, } d(M, S)=\max _{\mathrm{i}}\lfloor\mathrm{Mi}-\mathrm{Si}\rfloor
$$

Based on the definition of the proximity, different classification methods are reported in the literature including Nearest Neighbor Classification. Further, Nearest Neighbor classification itself is decimated into the successful implementation of three types of classifiers including Nearest Neighbor Classifier, k-Nearest Neighbor Classifier and Minimum Mean Distance Classifier [1-4] as per the following details.

\subsection{Nearest Neighbor Classifier}

Nearest neighbor decision rule assigns to an unclassified sample point as shown in Figure 1. This rule is independent of the underlying joint distribution on the sample points and their classifications. For predicting the class of a test example nearest-neighbor method is perhaps the simplest of all algorithms. During training phase, simply store every training example, with its label. To make a prediction for a test example, the method computes its distance to every training example. The distance of an input feature vector $M$ of unknown class to a class, $C_{j}$, is defined as the distance to the closest sample that is used to represent the class [2].

$$
d\left(M, C_{j}\right)=\min _{i} d\left(M, M_{i}^{j}\right)
$$

where, $d\left(M, M_{i}^{j}\right)$ is the distance between $M$ and $\mathrm{M}_{i}{ }^{j}$. A pattern $\mathrm{M}$ of unknown classification to the class of its nearest neighbor is assigned by classification rule. $M \in$ Class $C_{j}$, if $d\left(M, C_{j}\right)=\min _{i} d\left(M, C_{j}\right)$ 


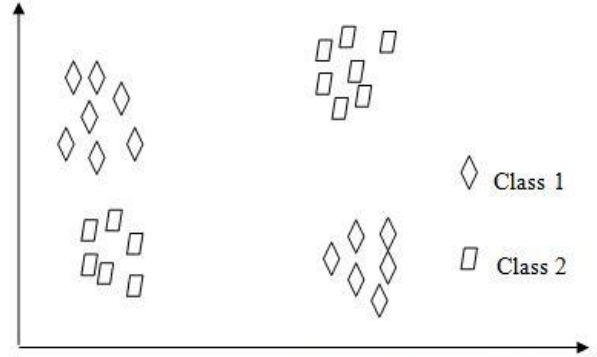

Fig1: Graphical Representation of Nearest Neighbor Classification

\section{2 k- Nearest Neighbor Classifier}

In k-nearest neighbor classification, an input feature vector $\mathrm{M}$ is classified into class $C_{\mathrm{j}}$, based on a voting mechanism as shown in Figure 2. Among all of the classes the classifier finds the k-nearest samples. The input feature vector of the unknown class is assigned to the class with the majority of the votes in the k-nearest samples. When there is no or little prior information about the distribution of the data then k-nearestneighbor ( $\mathrm{k}-\mathrm{NN}$ ) classification is one of the most preferred classification methods. k-Nearest Neighbor classification is more robust to noise comparison with Nearest Neighbor Classification [1-4].

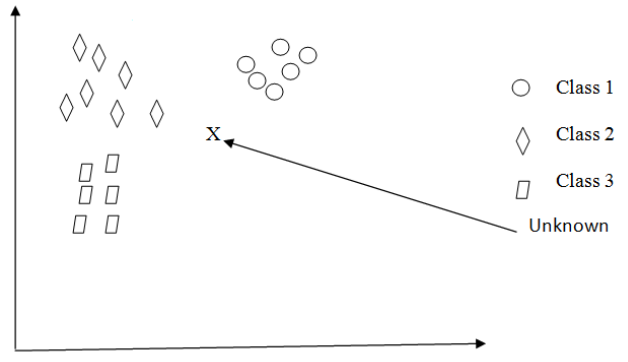

Fig.2: Graphical Representation of k-Nearest Neighbor Classification

\subsection{Minimum Mean Distance Classifier}

The feature patterns of each class tend to cluster tightly around the class center in applications that have no to less feature variability pattern or more noise is present. Under these available conditions, Minimum Mean Distance classifiers perform effectively. In this case, only the input vector distances to the centers of the classes need to be calculated instead of all the representative samples in realtime classification. Let $\left\{X^{j}{ }_{1}, X^{j}{ }_{2}, \ldots, X_{n j}^{j}\right\}$ be $n_{j}$ feature vectors that represent class $C_{j}[2]$. Each feature vector has the label of class $j$ that has to be selected to represent the class. The center of the class $j$ is defined as

$$
M_{j=} \frac{1}{n_{j}} \sum_{i=1}^{n_{j}} X^{j}
$$

The classification phase classifies an input feature vector $X$ of unknown class based on its distance to each class center.

$$
X \in \operatorname{class} C_{j} \text {, if } d\left(X, M_{j}\right)=\min d\left(X, M_{j}\right)
$$

where, $d\left(X, M_{j}\right)$ is defined as the distance function based on the distance metric selected during the training phase [2].

\section{EXPERIMENTAL SETUP}

Experimental setup used for the purpose of experimental validation of the proposed intelligent estimator is illustrated in Figure 3 . The visual based color classification system mainly employs simple input device and software. The performance of the implemented intelligent estimator is examined experimentally for assessing quality of red delicious apple using industrial grade BASLER sca-1390 17fc color camera connected to an image grabber of a computer based machine vision system. Image acquisition card, camera and software are executed on a computer. NI Vision Builder for Automated Inspection is chosen for the development of machine vision software in LabVIEW $\mathrm{v} 8.6$ environment as it provides excellent facility for the implementation of suitable graphical user interface (GUI). The image taken is digitized as 24-bit RGB data at the resolution of $1390 \times 1038$. Data is then processed and analyzed. The implemented color classification training interface is used to train a color classifier by manually classifying color samples into new or existing color classes. The executed color classifier calculates the color features in which the color sample is first converted from RGB to HSL color space. Then hue, saturation and luminance histograms of the color sample are computed. The hue and saturation histograms each contain 256 values whereas luminance histogram is reduced to 8 values thereby achieving $12.5 \%$ total suppressing. By suppressing the luminance histogram, the executed color classifier accentuates the color information for the sample. In the last, the 520 hue, saturation and luminance values are combined to produce a high resolution color feature. Then a dynamic mask is applied to obtain a medium resolution color features from high resolution color features. In fact, the medium resolution color features are subsets of high resolution color feature containing 128 hue and saturation values and 8 luminance values for a total of 136 values. Medium resolution feature is selected to speed up color classification process in the proposed work. The classification process is responsible to classify the input or user selected fruit by using classifier algorithm. This measure the distance between features values of the stored fruit test.

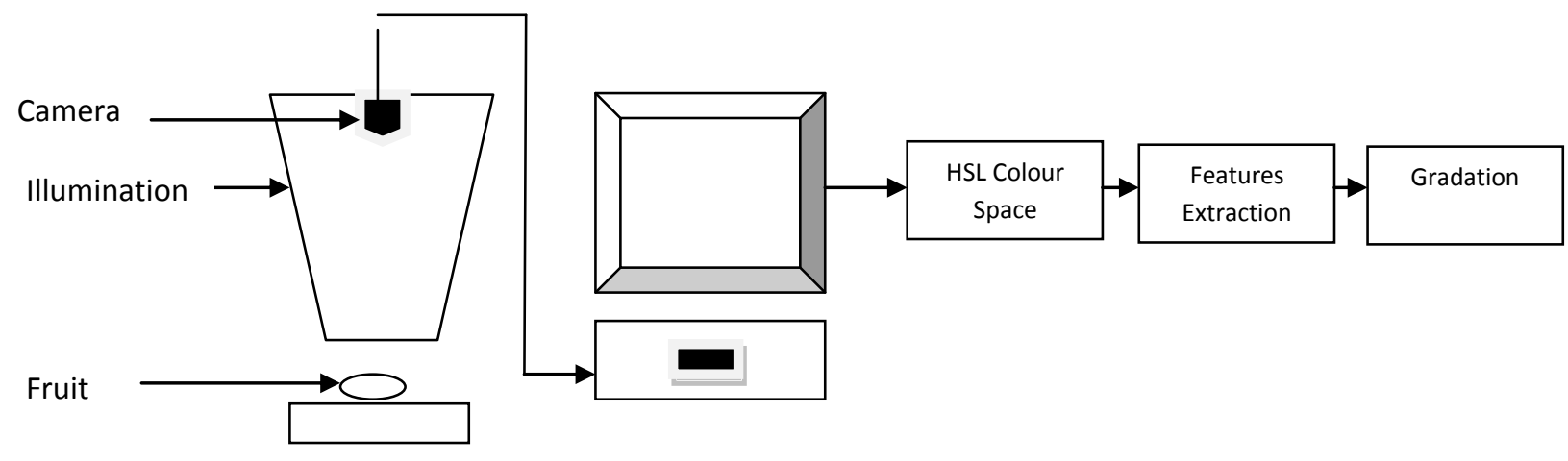

Fig.3: Experimental Setup 


\section{EXPERIMENTATION}

Two hundred apples of red delicious variety were taken for experimentation purpose. Three human experts trained in the field of grading of this particular variety were given the samples. They graded the apples on the basis of CODEX Stan 299 standard for apples which says that apples must have red color content [19]. So, four grades A, B, C and D with 25 samples in each were selected based on red color content. Fifteen samples in each grade were taken that were commonly agreed upon by all the experts. These were fed to the classifier for training. The inspection image contains multiple samples. The region of interest toolbar is used to define a region which is useful for training. The Annulus ROI tool is chosen for apple because it adjusts the inner and outer radii, and also adjusts the start and end angles. Training and classifying phases are there in a color classifier. During the training phase, the classifier is provided with 15 samples in each grade, i.e., A, B, C and D. A known sample consists of an area in the image, containing the color the classifier needs to learn. A color feature is calculated by the classifier and associated class label is assigned to the feature for every sample that is included in the training phase. Eventually, all the trained samples added to the classifier are saved into a file which represents a trained color classifier. After training the classifier, regions are classified into their corresponding classes for color identification in the apple quality. In this classifying phase, the classification engine calculates the color feature of the sample that need to be identified and classify it among trained sample using k-Nearest Neighbor Algorithm.

\section{RESULTS AND DISCUSSION}

Experimentation has been conducted using database of sixty training samples selected by three human experts with fifteen samples in each category. Using these training samples, three different types of engine methods were trained. These engine methods include nearest neighbor engine, k-nearest neighbor engine and minimum mean distance engine. Each engine is executed using three distance metrics including Sum (Manhattan), Euclidean and Maximum Distance. In the testing phase, 100 test samples selected by human experts with 25 samples in each category were given to the proposed intelligent estimator. The estimator classifies and grades them according to their color content. The performance of each variant is examined by varying the number $(\mathrm{k})$ of training sample from 3 to 15 as well as illumination intensity. The percentage accuracy achieved using these nine different types of variants of nearest neighbor algorithm, k-nearest neighbor algorithm and minimum mean distance algorithm is indicated in Table-1, Table-2, Table-3, Table-4 and Table-5 for five different values of surface illumination. It has been found experimentally that k-Nearest Neighbor based classifier using Euclidean distance metric gives highest accuracy of $88.61 \%$ ,91.25\%, 95.12\%, 92.07\% and $85.63 \%$ at illumination intensity of 486 Lux, 405 Lux,310 Lux, 253 Lux and 170 Lux, respectively with number of training samples chosen as 10 , $12,11,10$ and 7 , respectively. Based on this observation, it is very much evident that illumination intensity plays an extremely important role in determining the efficiency and accuracy of the system. Further, it is also found experimentally that the proposed intelligent estimator gives highest efficiency of $95.12 \%$ when trained with 11 numbers of training samples at an illumination intensity of 310 Lux assuming manual grading to be $100 \%$ as reference level. The results of this study are also summarized in Table-6. Thus, it has been found experimentally that number of training samples chosen and choice of fruit surface illumination are two important parameters which must be taken care of while designing the proposed k-nearest neighbor based intelligent estimator.

It has also been found experimentally that the reasonable amount of accuracy is achievable using medium resolution color feature containing 128 hue and saturation values and 8 luminance values making a total of 136 values. The present study, thus, confirms that the proposed intelligent estimator estimates fruit quality with considerable accuracy using machine vision exhibiting at the same time an effective human visual perception. In fact, the results of this study are quite promising. The results exhibited by the implemented estimator are further validated by doing manual grading of the nearly $4.88 \%$ fruit wrongly graded by the proposed estimator ten times. It has been found that out of $4.88 \%$ apple wrongly graded by the estimator are also graded wrongly to the level of nearly $4.4 \%$ by the human experts thus confirming subject perception of human vision. Further, during introspection, the human experts confirm that nearly $4.88 \%$ apple are the border cases with regard to different classes and hence as such it is not possible to make a firm distinction manually on such type of samples. However, when the same samples are validated by executing the intelligent estimator, the repeatability is found to be again $100 \%$.

\section{CONCLUSION}

It has been found experimentally that k-Nearest Neighbor Classifier employing Euclidean Distance Metric estimates quality of red delicious apple with considerable accuracy when fruit surface is illuminated at an optimal level of intensity. Moreover, the proposed estimator when trained with an optimal number of training samples achieves an accuracy of nearly $95.12 \%$ if manual grading is assumed to be $100 \%$ accurate taken as reference level. However, after having rigorous experimental validation, the repeatability of the proposed system is found to be $100 \%$. The results obtained in the real time operation of the proposed intelligent estimator confirms the results obtained in the training and classifying phases. The theoretical principles and practical design of the proposed intelligent estimator are described. The technique would be quite useful for other types of fruits possessing similar surface characteristics. In fact the proposed technique has a potential future in the field of machine vision based inspection of agriculture produce. 
Table-1. Percentage Accuracy at Illumination Intensity of 486 Lux

\begin{tabular}{|c|c|c|c|c|c|c|c|c|c|}
\hline \multirow{2}{*}{$\begin{array}{c}\text { Number of Training } \\
\text { Samples stored in Classifier } \\
(\mathrm{k})\end{array}$} & \multicolumn{2}{|c|}{ Nearest Neighbor Classifier } & \multicolumn{2}{|c|}{ k-Nearest Neighbor Classifier } & \multicolumn{2}{l|}{ Minimum Mean Distance Classifier } \\
\cline { 2 - 11 } & Sum & Euclidean & Maximum & Sum & Euclidean & Maximum & Sum & Euclidean & Maximum \\
\hline 3 & 49.10 & 56.20 & 56.03 & 55.96 & 64.37 & 31.12 & 55.11 & 61.45 & 39.64 \\
\hline 4 & 44.30 & 52.15 & 49.14 & 61.14 & 59.14 & 30.33 & 56.14 & 66.78 & 46.47 \\
\hline 5 & 39.11 & 54.63 & 51.17 & 48.14 & 60.12 & 24.78 & 61.44 & 68.14 & 50.15 \\
\hline 6 & 37.13 & 35.14 & 39.87 & 40.17 & 68.15 & 30.47 & 50.15 & 61.14 & 52.21 \\
\hline 7 & 29.14 & 29.25 & 28.21 & 31.33 & 59.14 & 38.96 & 52.77 & 64.60 & 62.21 \\
\hline 8 & 31.67 & 38.16 & 31.15 & 38.22 & 59.19 & 48.65 & 50.98 & 68.12 & 60.22 \\
\hline 9 & 41.54 & 47.14 & 41.25 & 50.24 & 69.67 & 61.75 & 58.14 & 70.31 & 67.11 \\
\hline 10 & 46.36 & 66.23 & 61.65 & 68.11 & 88.61 & 84.15 & 78.14 & 79.12 & 73.24 \\
\hline 11 & 51.93 & 76.58 & 64.21 & 77.16 & 81.14 & 79.78 & 77.26 & 72.14 & 60.55 \\
\hline 12 & 49.45 & 71.35 & 60.11 & 69.64 & 79.65 & 74.15 & 71.14 & 68.11 & 59.63 \\
\hline 13 & 46.63 & 69.18 & 59.06 & 64.15 & 71.14 & 69.11 & 69.26 & 61.10 & 55.22 \\
\hline 14 & 45.47 & 61.22 & 54.25 & 60.12 & 69.33 & 63.19 & 64.69 & 62.01 & 58.36 \\
\hline 15 & 39.27 & 54.78 & 49.12 & 55.25 & 64.44 & 55.33 & 59.14 & 60.25 & 55.11 \\
\hline
\end{tabular}

Table-2. Percentage Accuracy at Illumination Intensity of 405 Lux

\begin{tabular}{|c|c|c|c|c|c|c|c|c|c|}
\hline $\begin{array}{c}\text { Number of Training } \\
\text { Samples stored in Classifier } \\
(\mathrm{k})\end{array}$ & \multicolumn{2}{|c|}{ Nearest Neighbor Classifier } & \multicolumn{2}{l|}{ k-Nearest Neighbor Classifier } & \multicolumn{3}{l|}{ Minimum Mean Distance Classifier } \\
\cline { 2 - 10 } & Sum & Euclidean & Maximum & Sum & Euclidean & Maximum & Sum & Euclidean & Maximum \\
\hline 3 & 79.11 & 63.14 & 63.47 & 61.15 & 74.55 & 69.14 & 66.22 & 66.12 & 68.23 \\
\hline 4 & 78.14 & 51.15 & 58.12 & 69.45 & 68.66 & 69.69 & 65.44 & 59.11 & 64.15 \\
\hline 5 & 71.33 & 55.69 & 63.17 & 55.14 & 66.55 & 55.25 & 46.14 & 52.55 & 64.11 \\
\hline 6 & 58.16 & 58.66 & 57.19 & 54.11 & 81.22 & 64.14 & 59.11 & 71.58 & 59.58 \\
\hline 7 & 46.77 & 46.14 & 48.33 & 49.36 & 46.23 & 71.15 & 71.39 & 46.26 & 72.51 \\
\hline 8 & 51.98 & 56.18 & 48.21 & 52.15 & 61.21 & 56.11 & 64.18 & 51.26 & 52.51 \\
\hline 9 & 64.48 & 66.32 & 54.32 & 66.14 & 71.44 & 66.69 & 51.18 & 55.21 & 56.15 \\
\hline 10 & 76.31 & 53.15 & 59.25 & 64.11 & 84.14 & 76.36 & 64.56 & 74.48 & 74.11 \\
\hline 11 & 76.38 & 63.19 & 61.36 & 79.46 & 91.12 & 79.25 & 71.23 & 82.18 & 79.01 \\
\hline 12 & 72.21 & 66.77 & 59.17 & 72.64 & 91.25 & 75.14 & 68.36 & 75.18 & 71.36 \\
\hline 13 & 61.56 & 54.89 & 52.15 & 61.21 & 74.36 & 71.10 & 54.62 & 55.15 & 64.39 \\
\hline 14 & 62.65 & 59.39 & 51.36 & 59.31 & 74.14 & 71.09 & 51.45 & 50.02 & 64.31 \\
\hline 15 & 49.32 & 55.37 & 49.32 & 56.39 & 68.36 & 69.01 & 46.55 & 49.01 & 61.15 \\
\hline
\end{tabular}

Table-3. Percentage Accuracy at Illumination Intensity of 310 Lux

\begin{tabular}{|c|l|c|c|c|c|c|c|c|c|}
\hline \multirow{2}{*}{$\begin{array}{c}\text { Number of Training } \\
\text { Samples stored in } \\
\text { Classifier (k) }\end{array}$} & \multicolumn{3}{|c|}{ Nearest Neighbor Classifier } & \multicolumn{3}{c|}{ k-Nearest Neighbor Classifier } & \multicolumn{3}{c|}{$\begin{array}{c}\text { Minimum Mean Distance } \\
\text { Classifier }\end{array}$} \\
\cline { 2 - 10 } & Sum & Euclidean & Maximum & Sum & Euclidean & Maximum & Sum & Euclidean & Maximum \\
\hline 3 & 85.23 & 65.14 & 65.63 & 65.65 & 80.56 & 75.14 & 70.14 & 70.17 & 70.15 \\
\hline 4 & 81.32 & 56.65 & 62.36 & 75.14 & 75.32 & 75.55 & 68.36 & 62.15 & 68.14 \\
\hline 5 & 75.15 & 58.55 & 66.35 & 58.15 & 75.26 & 58.15 & 50.32 & 58.46 & 66.69 \\
\hline 6 & 62.25 & 62.15 & 62.31 & 62.14 & 87.12 & 68.54 & 62.39 & 75.51 & 62.45 \\
\hline 7 & 50.14 & 50.14 & 50.54 & 50.65 & 50.32 & 75.15 & 75.21 & 50.39 & 75.34 \\
\hline 8 & 55.48 & 60.46 & 52.14 & 57.60 & 65.15 & 60.12 & 65.15 & 55.28 & 55.18 \\
\hline 9 & 68.48 & 70.69 & 52.11 & 68.10 & 74.02 & 70.69 & 55.15 & 60.21 & 60.69 \\
\hline 10 & 79.15 & 55.59 & 62.15 & 70.03 & 87.02 & 80.69 & 60.15 & 78.99 & 78.18 \\
\hline 11 & 80.96 & 64.26 & 65.19 & 82.21 & 95.12 & 84.25 & 75.15 & 86.15 & 82.14 \\
\hline 12 & 76.18 & 67.25 & 63.99 & 76.12 & 85.25 & 79.12 & 71.11 & 79.18 & 76.99 \\
\hline 13 & 65.88 & 58.15 & 57.88 & 66.61 & 78.56 & 75.32 & 57.66 & 59.19 & 67.47 \\
\hline 14 & 66.58 & 62.15 & 55.15 & 63.35 & 77.36 & 76.12 & 56.68 & 55.88 & 68.17 \\
\hline 15 & 53.56 & 59.66 & 54.32 & 61.02 & 71.66 & 72.14 & 51.18 & 53.99 & 66.14 \\
\hline
\end{tabular}


Table-4. Percentage Accuracy at Illumination Intensity of 253 Lux

\begin{tabular}{|c|c|c|c|c|c|c|c|c|c|}
\hline $\begin{array}{c}\text { Number of Training } \\
\begin{array}{c}\text { Samples stored in } \\
\text { Classifier (k) }\end{array}\end{array}$ & \multicolumn{3}{|c|}{ Nearest Neighbor Classifier } & \multicolumn{3}{c|}{ k-Nearest Neighbor Classifier } & \multicolumn{3}{c|}{$\begin{array}{c}\text { Minimum Mean Distance } \\
\text { Classifier }\end{array}$} \\
\cline { 2 - 11 } & Sum & Euclidean & Maximum & Sum & Euclidean & Maximum & Sum & Euclidean & Maximum \\
\hline 3 & 50.69 & 58.69 & 58.18 & 57.90 & 66.21 & 58.98 & 58.58 & 66.81 & 50.69 \\
\hline 4 & 46.45 & 54.66 & 50.99 & 64.19 & 62.89 & 54.58 & 50.47 & 70.10 & 49.45 \\
\hline 5 & 40.44 & 56.15 & 54.18 & 50.03 & 62.80 & 56.47 & 63.14 & 72.05 & 54.14 \\
\hline 6 & 38.69 & 38.61 & 40.18 & 56.67 & 72.14 & 48.14 & 69.58 & 64.06 & 56.14 \\
\hline 7 & 30.48 & 30.15 & 32.18 & 57.78 & 70.15 & 50.89 & 67.58 & 68.04 & 65.11 \\
\hline 8 & 35.14 & 40.15 & 35.14 & 45.78 & 63.18 & 51.98 & 54.21 & 71.04 & 63.56 \\
\hline 9 & 45.39 & 50.11 & 45.18 & 47.13 & 75.98 & 65.48 & 60.96 & 74.96 & 69.87 \\
\hline 10 & 51.14 & 70.66 & 65.17 & 58.13 & 92.07 & 70.68 & 65.14 & 81.07 & 75.69 \\
\hline 11 & 55.58 & 80.69 & 68.36 & 54.67 & 85.66 & 80.48 & 68.48 & 56.03 & 55.21 \\
\hline 12 & 51.48 & 76.55 & 64.69 & 50.23 & 71.14 & 76.47 & 64.58 & 48.06 & 51.54 \\
\hline 13 & 47.15 & 70.48 & 62.15 & 43.05 & 69.87 & 70.85 & 62.64 & 67.05 & 47.64 \\
\hline 14 & 49.36 & 63.47 & 54.36 & 50.13 & 59.48 & 63.54 & 54.14 & 35.04 & 49.34 \\
\hline 15 & 41.21 & 58.12 & 50.21 & 40.21 & 51.69 & 58.69 & 50.63 & 56.78 & 41.14 \\
\hline
\end{tabular}

Table-5. Percentage Accuracy at Illumination Intensity of 170 Lux

\begin{tabular}{|c|l|l|l|c|c|c|c|c|c|}
\hline $\begin{array}{c}\text { Number of Training } \\
\begin{array}{c}\text { Samples stored in } \\
\text { Classifier (k) }\end{array}\end{array}$ & \multicolumn{3}{|c|}{ Nearest Neighbor Classifier } & \multicolumn{2}{c|}{ k-Nearest Neighbor Classifier } & \multicolumn{3}{c|}{$\begin{array}{c}\text { Minimum Mean Distance } \\
\text { Classifier }\end{array}$} \\
\cline { 2 - 10 } & Sum & Euclidean & Maximum & Sum & Euclidean & Maximum & Sum & Euclidean & Maximum \\
\hline 3 & 40.56 & 45.45 & 45.36 & 42.14 & 48.17 & 40.14 & 45.58 & 48.18 & 40.89 \\
\hline 4 & 45.21 & 50.04 & 54.15 & 48.14 & 56.18 & 44.58 & 48.11 & 52.18 & 48.14 \\
\hline 5 & 50.15 & 54.36 & 59.25 & 58.13 & 61.21 & 50.15 & 52.10 & 56.12 & 54.85 \\
\hline 6 & 60.15 & 59.90 & 66.36 & 69.12 & 72.12 & 56.14 & 59.78 & 61.14 & 60.14 \\
\hline 7 & 67.14 & 69.36 & 73.31 & 72.14 & 85.63 & 67.01 & 65.15 & 69.18 & 65.98 \\
\hline 8 & 70.99 & 72.16 & 75.31 & 76.15 & 80.19 & 63.36 & 62.69 & 61.22 & 59.14 \\
\hline 9 & 74.18 & 73.25 & 70.35 & 71.03 & 78.17 & 65.15 & 60.11 & 58.88 & 57.56 \\
\hline 10 & 76.15 & 70.11 & 68.15 & 69.21 & 76.20 & 67.11 & 58.18 & 56.17 & 55.14 \\
\hline 11 & 72.21 & 68.36 & 70.14 & 66.18 & 72.48 & 66.25 & 61.17 & 62.11 & 48.16 \\
\hline 12 & 69.45 & 65.25 & 66.25 & 64.15 & 70.14 & 62.15 & 58.18 & 62.02 & 51.28 \\
\hline 13 & 65.63 & 61.21 & 63.14 & 61.16 & 68.96 & 59.15 & 56.17 & 59.03 & 51.69 \\
\hline 14 & 64.14 & 59.15 & 61.25 & 58.48 & 65.35 & 57.14 & 54.98 & 56.17 & 49.41 \\
\hline 15 & 63.48 & 63.69 & 65.14 & 60.17 & 67.14 & 56.15 & 50.17 & 51.12 & 52.36 \\
\hline
\end{tabular}

Table-6. Performance Summary of the Proposed Intelligent Estimator

\begin{tabular}{|c|c|c|}
\hline $\begin{array}{c}\text { Optimal Number of Training } \\
\text { Sample }\end{array}$ & Optimal Surface illumination (Lux) & $\begin{array}{c}\text { Maximum Accuracy } \\
\text { Achieved }(\%)\end{array}$ \\
\hline 10 & 486 & 88.61 \\
\hline 12 & 405 & 91.25 \\
\hline 11 & 310 & 95.12 \\
\hline 10 & 253 & 92.07 \\
\hline 7 & 170 & 85.63 \\
\hline
\end{tabular}

\section{REFERENCES}

[1] http://zone.ni.com/reference/enXX/help/372916 M01/nivisionconcepts/classification_methods/.

[2] NI Vision Concepts Manual, August 2009

[3] NI Vision Builder for Automated Inspection Manual, August 2009.

[4] Jeffrey Travis and Jim Kring, "LabVIEW for Everyone Graphical Programming made easy an Fun" $\left(2009^{\text {th }}\right.$ Edition), Pearson Education.
[5] Rafael C. Gonzalez and Richard E. Woods, "Digital Image Processing", ( $3^{\text {rd }}$ Edition), Pearson Education.

[6] Anil K Jain, "Fundamentals of Digital Image Processing" , ( $3^{\text {rd }}$ Edition), Prentice Hall Edition

[7] Ling Mei Chan, Rodney Tan and Gilbert Thio, "Design of Visual Based Color Classification System", JASA 2 (January 2007),30-33.

[8] Mohd Hafiz MohdHazir and Abdul Rashid Mohamed Shariff, "Oil Palm Optical Characteristics from Two Different Planting Materials". 2011 International Conference on Future Information Technology IPCSIT 
vol.13, 196-200.

[9] D.J. Lee, Yuchou Chang, James K. Archihald Chrisopher G. Greco, "Color Quantization and Image Analysis for Automated Fruit Quality Evaluation", $4^{\text {th }}$ IEEE Conference on Automation Science and Engineering key Bridge, Marriot, Washington DC,USA (August 2008), 23-26.

[10] P.SudhakaraRao, A.Gopal, R. Revathy and K. Meenakshi "Colour Analysis of Fruits Using Machine Vision System for Automatic Sorting And Grading", J.Instrum.Soc.India 34(4), 284-291

[11] Yizhong Wang, Yanhua Chi, Huafang Huang, Shahui Chen, Ping Chang, George Q. Huang, "Study on HIS Color Model Based Fruit Quality Evaluation", $3^{\text {rd }}$ International Congress on Image and Signal Processing (CISP-2010) vol 6, 2677-2680.

[12] Balkrishan Ahirwal, Mahesh Khadtare and Rakesh Mehta, "FPGA based system for color space Transformation RGB to YIQ and YCbCr,"International Conference on Intelligent and Advanced systems (2007), 1345-1349.

[13] Young-Chang and John F. Reid, "RGB Calibration for Color Image Analysis in Machine Vision", IEEE Transactions on Image Processing, Vol 5, No. 10, (October 1996), 1414-1422.
[14] S. Arivazhagan, R. NewlinShebiah, S. elva Nidhyanandhan and L. Ganesan, "Fruit Recognition using Color and Texture Features". Journal of Emerging Trends in Computing and Information Sciences, Vol 1, No.2 (Oct 2010), 90-94.

[15] F. Guevara-Hernandez and J. Gomez-Gil "A Machine Vision system for classification of wheat and Barley grain Kernels". Spanish Journal of Agricultural Research 9 (3) (2011), 672-680.

[16] DevrimUnay, Bernard Gosselin, Olivier Kleynen, Vincent Leemans, Marie-France Destain and Olivier Debeir " Automatic Grading of Bi-Colored Apples by Multispectral Machine vision. Journal of Computers and Electronics in Agriculture, Elsevier (2010), 204-212.

[17] D S Guru, Y. H Sharath and S. Manjunath " Texture Features and KNN in Classification of Flower Images" IJCA Special Issue on Recent Trends in ImageProcessing and Pattern Recognition (RTIPPR 2010), 21- 29.

[18] T. M Cover, Member, IEEE, and P. E. Hart, Member, IEEE, "Nearest Neighbor Pattern Classification.

[19] http://www.codexalimentarius.org/input/download/standa rds/11509/CXS_299e.pdf 